\title{
Laparoscopic Damage Control in Type IV Paraesophageal Hiatal Hernia after Minimally Invasive Esophagectomy: Case Report
}

\author{
Evelyn Astrid Dorado ${ }^{1^{*}}$, Jessica Correa ${ }^{2}$, Keyla Castillo ${ }^{3}$ and Hernán Esteban Munevar \\ ${ }^{1}$ Department of Minimally Invasive Surgery and Bariatric Surgery, Fundación Valle del Lili, Universidad ICESI, Colombia \\ ${ }^{2}$ Department of Surgery, Fundación Valle del Lili, Colombia \\ ${ }^{3}$ Faculty of Health Sciences, Complejo Hospitalario Doctor Arnulfo Arias Madrid, Panama \\ ${ }^{4}$ Clinical Research Center, Fundación Valle del Lili, Cali, Colombia
}

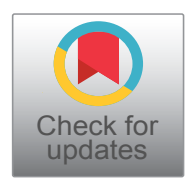

* Corresponding author: Dr. Evelyn Astrid Dorado Alban, FACS, FASMBS, Chief, Department of Minimally Invasive Surgery and Bariatric Surgery, Fundación Valle del Lili, Associte Professor Universidad ICESI, Fundación Valle del Lili, Av. Simón Bolívar Carrera, 98 \#98-49, Cali, Colombia, Tel: (572)-3319090, Ext: 4022, Fax: (57)-(2) +315-79-74, ORCID iD: 0000-00025986-524X

\begin{abstract}
Post-esophagectomy hiatal hernia is an uncommon condition. Patients may present with small asymptomatic hiatal hernias, nonspecific chronic symptoms or even acute presentations with signs of intestinal obstruction and ischemia. In this case, a three-stage esophagectomy was performed in a female patient diagnosed with end-stage achalasia with subsequent development of hiatal hernia strangulation, for which damage control surgery was key for its management. The implication of damage control surgery in treating strangulated hiatal hernias is an uncommon but valid occurrence as an option for the surgical management within the clinical context of critically ill patients.
\end{abstract}

\section{Keywords}

Hiatal hernia, Laparoscopic esophagectomy, Damage control surgery

\section{Background}

The occurrence of post-esophagectomy hiatal hernia is a rare condition that is being increasingly diagnosed. The acute presentation with signs of obstruction and strangulation may be life-threatening and involve intestinal resections with increased mortality rates $[1,2]$. This review is reporting a case that required the use of damage control surgery within the surgical strategies for managing an incarcerated hiatal hernia case report. This 68-year-old female patient has a history of acha- lasia that required myotomy by open chest approach at age 39. Three years ago, she started with cough, regurgitation and mild dysphagia for which the following paraclinical tests were requested: Barium-swallow studio showed esophageal dilatation with distal bird beak pattern and delayed emptying at 2 hours (Figure 1A). An endoscopy informed very tortuous esophagus with retained food; the gastroesophageal junction was easily passed through; hiatal and antral hernia with retention; and possible vagotomy. The manometry reported normal upper esophageal sphincter; lack of pressure at the lower esophageal sphincter and nonpropulsive esophageal body. CT-Scan showed esophageal distention with retained food; and simple hepatic cyst. Her medical history included arterial hypertension, COPD, and her surgical history included cesarean surgery, open appendectomy, laparoscopic cholecystectomy and rhinoplasty. No abnormal findings were observed during the physical exam. The patient was scheduled for a threestage minimally invasive esophagectomy and gastric pull up in the prone position. Firstly, in prone position with selective intubation, three trocars (two 5-mm and one 12-mm trocars) were inserted at the right subscapular level; an advance bipolar was used to release the pulmonary ligament, then the azygos vein was identified and dissected by ligation with hemo-locks; dissection of the intrathoracic esophagus was initiated $2 \mathrm{~cm}$ 


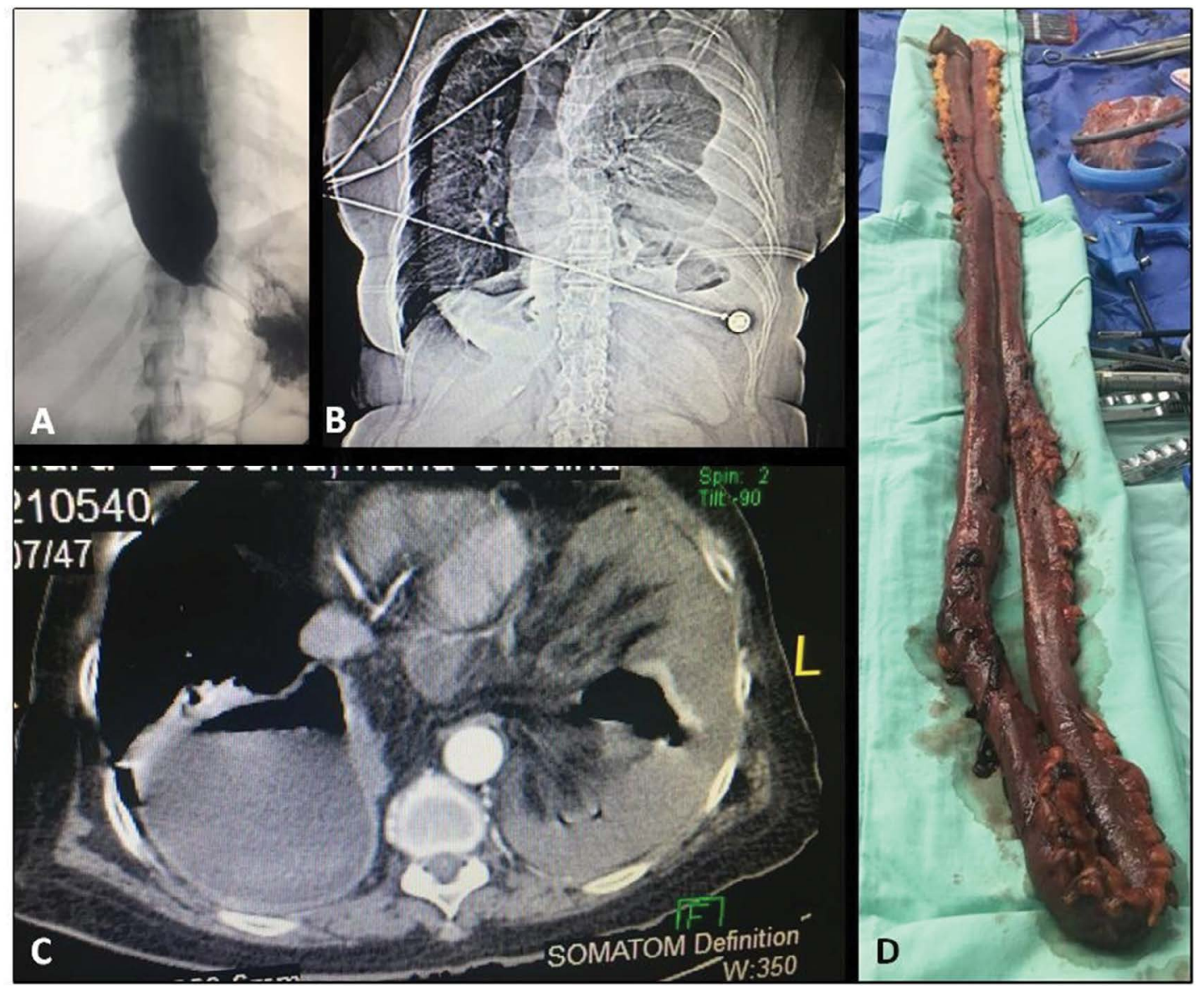

Figure 1: A) Fluoroscopy showing a large esophageal dilatation with distal bird beak pattern and delayed emptying at 2 hours; B) Chest X-ray showing small bowel loops within the left hemithorax and left costophrenic angle blunting; C) Computerized axial tomography showing collapse of the right lung and incarceration of small bowel loops into the left hemithorax; D) Resected small bowel segment sized $150 \mathrm{~cm}$.

above the azygos vein down to the diaphragmatic crura completing the thoracic stage. The patient was put in supine- French position and a right thoracic tube was placed. During the second stage, 5-trocar technique, an advance bipolar was used to release the greater curvature, sparing the gastroepiploic artery with dissection progressing to the left crura and dissection of the gastrohepatic membrane. Identification and ligation of the left gastric artery and vein with hemolocks, and dissection and release of the esophagus through the hiatus was then performed to complete the thoracic dissection. At the level of the right gastric vessels and in the direction to left shoulder, staples were applied with two $60-\mathrm{mm}$ blue cartridge without total gastric transection. At this point, the gastric tube was ready for pull up. During the third stage, left cervicotomy with dissection of the cervical esophagus was performed cautiously preventing injuries to the recurrent nerve of the larynx; video-assisted pull up of the gastric tube was performed preventing any torsion. Per protocol, the hiatus was not enlarged to avoid subsequent herniation. Finally, at the cervical level, resection of the esophagus was performed with a $60-\mathrm{mm}$ blue cartridge under the gastroesophageal junction and latero-lateral gastroesophageal anastomosis was carried out using a $60-\mathrm{mm}$ blue reload. A nasoenteral tube was inserted into the gastric tube and a cervical drainage was put in place. The patient was transferred extubated to the intensive care unit for postoperative management. After 24 hours, enteral nutrition at $5 \mathrm{cc} /$ hour was started through the nasogastric tube. On postoperative day 3 , an esophagogram that was performed showed anastomosis integrity and clear fluids were started with good tolerability. The patient was discharged on postoperative day 13 . The average stay for patients undergoing esophagectomy with video-assisted gastric ascension ranges from 5 to 8 days; the hospital stay for this patient was longer because of her underlying pulmonary disease requiring management with respiratory therapy. The patient continued in controls for 9 months, tolerating the soft diet, living a normal life.

Although she developed sudden breathing difficulty with ventilatory failure required transfer to a near clinic and urgent intubation; chest X-rays and tomography were performed, showing right side the ascended gastric tube and, on the left side of the chest, small pleura effusion with incarceration of small intestine loops and wall thickening were observed (Figure 1B and Figure 1C). Three days after the acute episode, the patient was referred to Fundación Valle de Lili Clinic for management by the department of minimally invasive surgery, the patient enters with orotracheal intubation 
to ICU, the CT-Scan showed s incarceration of small intestine loops through hiatus, small pleural effusion in the left side and gastric tube in the right side. Patient was scheduale for urgent laparoscopy, 5 trocars technique, $12 \mathrm{~mm}$ umbilical port and left paraumbilical, and $5 \mathrm{~mm}$ ports right paraumbilical, left border costal and subxiphoideo. There is a double loop of small intestine entering through the hiatus, which is not dilated and with intestinal ischemia. A $0.5-\mathrm{cm}$ incision was made on the hiatus and the small bowel loops were reduced in the left hemithorax; approximately $150 \mathrm{~cm}$ of necrotic small bowel were removed (Figure 1D) and the left hemithorax was washed using one liter of normal saline. The hiatus was closed using non-absorbable monofilaments and, given the absence of a giant hiatal hernia, placement of a biological mesh was not considered. Small isquemic segment recovered, but in the part with necrosis there were 2 perforations and part of the ischemic intestine did not recover, so it was necessary to do a resection of $150 \mathrm{~cm}$ of small intestine. During bowel resection the patient became hypotensive, acidotic, and it was necessary to use high doses of vasopressors, for this reason it was decided to perform a laparoscopic damage control surgery, stapling the intestine segments and transfer to ICU for resuscitation and revision in 36 to 72 hours of the stapled segments.

After 30 hours, the patient was stable with no vasopressor requirement and without acidosis, for which she was scheduled for second view and laparoscopic intestinal. The patient was extubated after the procedure and oral intake 24 hours later was well tolerated; the patient was discharged 5 days later on a liquefied diet and ambulatory oxygen therapy that was withdrawn one month after surgery.

\section{Discussion}

Achalasia is the result of the progressive degeneration of ganglion cells in the myenteric plexus of the esophageal wall that leads to loss of relaxation of the lower esophageal sphincter, in addition to loss of distal esophageal peristalsis. The characteristics of late- or end-stage achalasia include esophageal tortuosity, angulation and severe dilatation of megaesophagus (diameter greater than $6 \mathrm{~cm}$ ). Approximately $10-15 \%$ of patients undergoing achalasia treatment will develop late-stage achalasia, and up to $5 \%$ of patients in some series require esophagectomy [1]. Studies have shown that minimally invasive esophagectomy has a lower rate of blood loss, reduced hospital stay and overall decrease in morbidity $[2,3]$.

Esophagectomy is a surgical procedure that is performed for treating benign and especially malignant esophageal conditions. This procedure involves dissection and removal of the esophagus with restauration of the alimentary tract; as a result, the usual anatomy of the esophageal hiatus changes with increasing risks of developing post-esophagectomy hiatal hernia $[1,3]$.
Despite this, post-esophagectomy hiatal hernia is a rare complication. Its actual incidence may be underestimated and the survival of patients after esophagectomy has increased its occurrence. A recent meta-analysis reported that symptomatic hiatal hernias are more common following minimally invasive esophagectomy as compared to open procedures, with an incidence of $4.5 \%$ and $1.0 \%$, respectively, and a mean of $2.5 \%$ [3].

By definition, Hiatal Hernia the hiatal hernia can be classified into IV types, type I is when the esophagogastric junction is ascend to the thorax, type II the esophagogastric junction is in place but the fundus of the stomach is herniated, III the most common which is the combination of the type I and II and type IV occurs when and intraabdominal organ migrates cephalically to the mediastinum through the esophageal hiatus (colon, spleen, pancreas, bowel) $[3,4]$.

Symptoms associated with post-esophagectomy hiatal hernia may present early or late. Early presentation is related to the small adhesions occurring after the laparoscopic procedure; late presentation is associated with the progressive enlargement of the esophageal hiatus, the suction effect caused by the negative intrathoracic pressure and the increased intra-abdominal pressure [2]. Since patients may be asymptomatic or experience nonspecific symptoms, diagnosis may be difficult; in such cases, chest X-rays and tomographic scans usually confirm the diagnosis [4]. In acute presentations, the most common symptoms are difficulty breathing $(40 \%)$, vomiting $(40 \%)$, abdominal pain $(37 \%)$, dysphagia (11\%), chest pain (20\%) and constipation (20\%); likewise, obstruction and incarceration of the herniated organ may occur $[1,4]$. The contents reported for hiatal hernia include colon $(82 \%, 86 \%)$, small intestine $(19 \%, 46 \%)$, pancreas $(13 \%, 3 \%)$, liver $(7 \%)$ and spleen $(2 \%, 3 \%)$, which occur especially on the left side of the chest $(97 \%)[1,3,4]$. With regards to the postoperative time in which the hiatal hernia presents as a surgical urgency, the first year following esophagectomy has been reported as the most common; however, it may occur after one year [4]. In a literature review of 9 studies, the median time interval between esophagectomy and the diagnosis of hiatal hernia has recently been reported as 12 months (range: 1-32 months) [3].

In a study published in 2017, conservative treatment was reported successful in $90 \%$ of asymptomatic patients, with only 1 asymptomatic patient (7\%) in the study, who required urgent intervention; however, the evidence for justifying conservative versus surgical management in asymptomatic patients is lacking [1]. In fact, some authors prefer surgical management in asymptomatic patients because of the mortality and morbidity associated to acute presentations with intestinal obstruction and ischemia [3]. As for patients with an acute presentation with signs and symptoms of obstruction, ischemia or perforation of the herniated organ, urgent 
surgical management is the treatment of choice, with high mortality rates reported of $14.3 \%, 19 \%$ and $20 \%$, respectively $[1,3,4]$. In the case of this patient, her clinical presentation required urgent surgical management.

The repair of post-esophagectomy hiatal hernia by the minimally invasive approach has increased. There is no evidence that a specific technique may reduce the risk of developing hiatal hernia. However, some authors have made a number of recommendations such as leaving the pillars intact, closing the hiatus in its anterior aspect, fixating the gastric conduit to the diaphragmatic crura, using a biological mesh to close the defect, or making relaxation incisions in the diaphragm when a mesh is to be used [3].

In serious cases presenting with generalized peritonitis and septic shock where the compromised patient's normal physiology precludes a definitive and safe surgical procedure, with possible failure of the anastomosis or anatomic reconstruction, which is unlike to be tolerated by a critically ill patient [5]. In such cases, the patient benefits from damage control surgery. The translation of the principles for damage control in non-trauma emergencies is simple in terms of practical application. The main objective is to achieve patient stabilization for optimal results of the definitive management. In this review, no literature reviews were found regarding any other case in which damage control surgery is per- formed for the management of post-esophagectomy hiatal hernia with intestinal ischemia. In the case of this patient, her clinical condition prevented definitive management in one surgical time and satisfactory results were obtained by laparoscopic damage control surgery.

\section{Conflicts of Interest}

No conflict of interest is declared by the authors.

\section{References}

1. Brenkman H, Parry K, Noble F, van Hillegersberg R, Sharland D, et al. (2017) Hiatal hernia after esophagectomy for cancer. Ann Thorac Surg 103: 1055-1062.

2. Vallböhmer D, Hölscher AH, Herbold T, Gutschow C, Schröder W (2007) Diaphragmatic hernia after conventional or laparoscopic assisted transthoracic esophagectomy. Ann Thorac Surg 84: 1847-1852.

3. Benjamin G, Ashfaq A, Chang YH, Harold K, Jaroszewski D (2015) Diaphragmatic hernia post-minimally invasive esophagectomy: A discussion and review of literature. Hernia 19: 635-643.

4. Matthews J, Bhanderi S, Mitchell $H$, Whiting J, Vohra R, et al. (2016) Diaphragmatic herniation following esophagogastric resectional surgery: An increasing problem with minimally invasive techniques?: Post-operative diaphragmatic hernias. Surg Endosc 30: 5419-5427.

5. Weber DG, Bendinelli C, Balogh ZJ (2014) Damage control surgery for abdominal emergencies. Br J Surg 101: 109118. 